\title{
THE ORIGIN OF RADIO SCINTILLATION IN THE LOCAL INTERSTELLAR MEDIUM
}

\author{
JefFrey L. Linsky, ${ }^{1}$ Barney J. Rickett, ${ }^{2}$ AND SETH REDField ${ }^{3,4}$ \\ Received 2007 September 17; accepted 2007 November 2
}

\begin{abstract}
We study three quasar radio sources (B1257-326, B1519-273, and J1819+385) that show large-amplitude intraday and annual scintillation variability produced by the Earth's motion relative to turbulent-scattering screens located within a few parsecs of the Sun. We find that the lines of sight to these sources pass through the edges of partially ionized warm interstellar clouds where two or more clouds may interact. From the gas flow vectors of these clouds, we find that the relative radial and transverse velocities of these clouds are large and could generate the turbulence that is responsible for the observed scintillation. For all three sight lines the flow velocities of nearby warm local interstellar clouds are consistent with the fits to the transverse flows of the radio scintillation signals.
\end{abstract}

Subject headings: ISM: atoms — ISM: clouds — ISM: structure — line: profiles — ultraviolet: ISM — ultraviolet: stars

\section{OBSERVATIONS OF FAST RADIO SCINTILLATION}

Interstellar scintillation (ISS) is the apparent variation in flux density of very compact radio sources due to propagation through the irregular refractive index of the ionized interstellar medium. The phenomenon was discovered and investigated through its $100 \%$ variations in pulsar signals seen on timescales from minutes to days. Most extragalactic radio sources have angular diameters much too large to scintillate, in the same way that the angular size of planets suppresses their atmospheric twinkling. However, the most compact parts of some active galactic nuclei do fluctuate at a level of $1 \%-10 \%$ over times shorter than a few days in a phenomenon called intraday variation (IDV) (see Heeschen et al. 1987; Quirrenbach et al. 1992). Although this was initially thought to be intrinsic variation of the sources interpreted as due to emission from regions with brightness temperatures very much higher than the inverse Compton limit, it was subsequently explained as ISS somewhere within the $1 \mathrm{kpc}$ thick layer of the "warm ionized medium" (WIM) of our Galaxy, and implied maximum source brightness temperatures near the inverse Compton limit (e.g., Rickett et al. 2006).

However, among intraday variables, a few sources stand out as having unusually fast high-amplitude variations at frequencies of 3-8 GHz. With timescales less than a few hours, they have been called "intrahour variables" (IHV), the best studied of which are B1257-326, B1519-273, and J1819+385. Their short timescales can only be explained by scintillation in a local region whose electron density has much greater rms variation than in the general WIM. The distances to the scattering are estimated to be in the range 1-30 pc. However, until now no specific site in the local interstellar medium (ISM) has been identified as responsible. A common feature of the IHV observations is that there is a systematic annual variation in their timescales due to a changing velocity of the Earth relative to the interstellarscattering medium. Indeed, several observers have estimated the

\footnotetext{
1 JILA, University of Colorado and NIST, Boulder, CO 80309-0440; jlinsky@ jila.colorado.edu.

2 Department of Electrical and Computer Engineering, University of California San Diego, La Jolla, CA 92093-0407; bjrickett@ucsd.edu.

3 Department of Astronomy and McDonald Observatory, University of Texas, Austin, TX 78712-0259; sredfield@astro.as.utexas.edu.

4 Hubble Fellow.
}

characteristic transverse velocities of the medium relative to the Sun needed to fit the ISS observations (Bignall et al. 2006; Dennett-Thorpe \& de Bruyn 2003; Jauncey et al. 2003).

It is also interesting that only a very few sources varied on hourly timescales in the $5 \mathrm{GHz}$ VLA survey of 525 compact flatspectrum active galactic nuclei, even though about half of them varied at a low level on times of several days (Lovell et al. 2007). Thus the regions responsible for IHV cover a very small fraction of the sky. The basic goal of this paper is to compare the velocities from the rapid ISS sources with the velocities of the warm local interstellar clouds identified by Redfield \& Linsky (2008) and so find the regions of the local ISM responsible for the IHV. We find that the turbulence responsible for the scintillation may be generated by cloud-cloud interactions that could also enhance the ionization at cloud interfaces.

\section{WARM CLOUDS IN THE LOCAL INTERSTELLAR MEDIUM}

The Sun is located in a $\sim 100 \mathrm{pc}$ radius volume devoid of dense interstellar material known as the Local Bubble (Lallement et al. 2003). Within the Local Bubble, a group of warm partially ionized clouds is found in the immediate ( $\lesssim 20 \mathrm{pc})$ vicinity of the Sun and is often referred to as the local ISM (LISM). This collection of gas is composed of distinct components, or clouds. This is demonstrated by the fact that LISM sight lines often exhibit multiple distinct absorbers, on average $\sim 1.7$ absorbers per sight line (Redfield \& Linsky 2004); therefore, any given line of sight typically has between 1 and 3 components. In addition, physical characteristics (e.g., temperature, metal depletion, etc.) and cloud kinematics are spatially correlated, that is, individual clouds have unique properties and dynamics.

Redfield \& Linsky (2008) used the largest LISM observational database, based on high spectral resolution ultraviolet and optical absorption measurements, to investigate the kinematical properties of the closest LISM clouds. They derived three-dimensional velocity vectors for 15 clouds, which all reside within $15 \mathrm{pc}$, including the local interstellar cloud (LIC) and Galactic (G) cloud, the two closest clouds, whose dynamics were first calculated by Lallement \& Bertin (1992) and Lallement et al. (1995). The 15 velocity vectors are roughly parallel, indicating that they share a common origin or driver, an issue discussed by Frisch et al. 

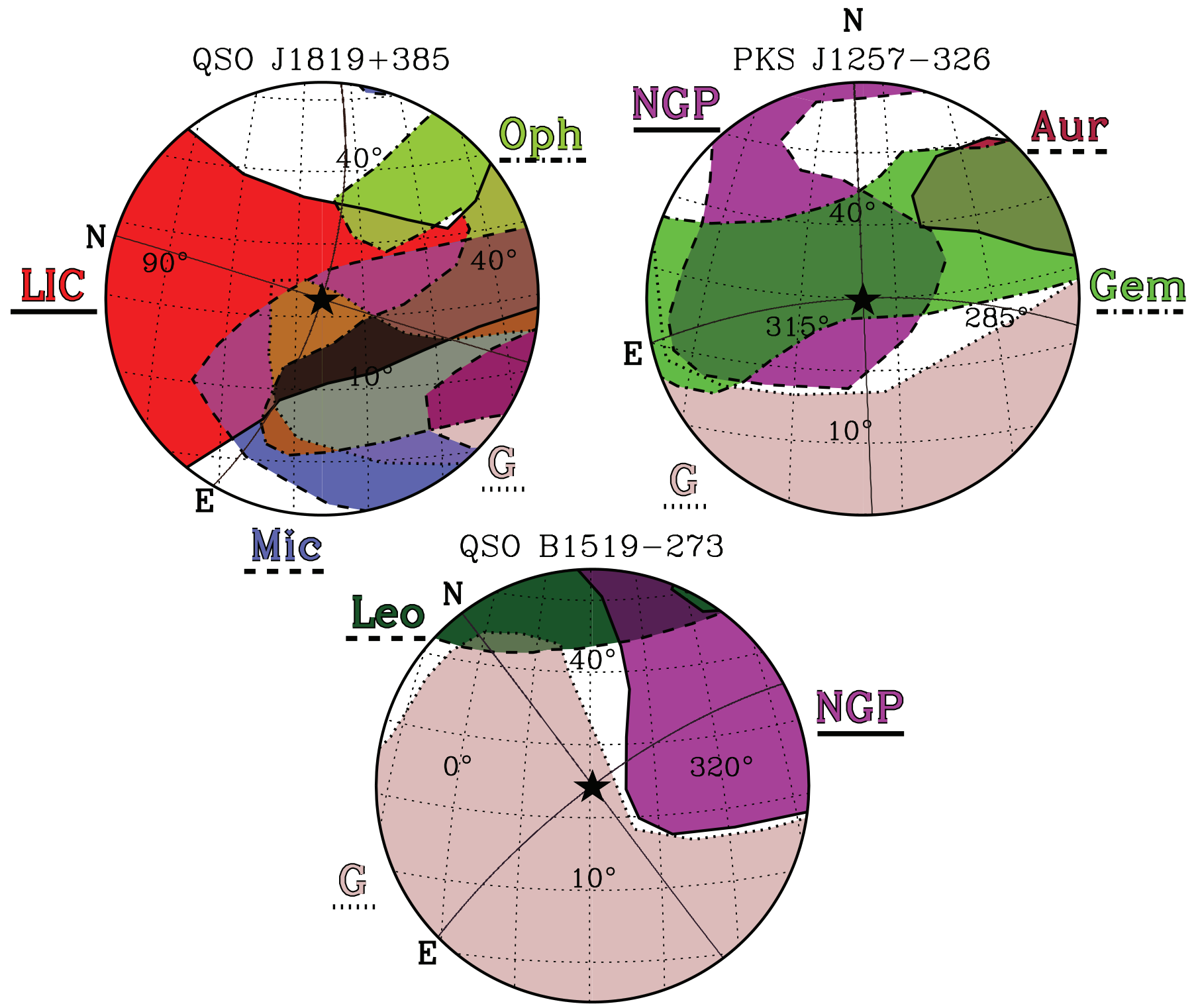

Fig. 1.- Morphology, in Galactic coordinates, of local interstellar clouds located within $30^{\circ}$ of the three quasars showing large-amplitude intraday and annular scintillation variability. The interstellar clouds identified by Redfield \& Linsky (2007) are color coded and labeled. Celestial north and east are identified in each plot.

(2002). However, the velocity vectors have a range of velocities $\left(0-50 \mathrm{~km} \mathrm{~s}^{-1}\right.$ relative to the local standard of rest), which implies that cloud-cloud collisions and interactions will play a prominent role in the physical properties of the LISM.

The outer edges of LISM clouds are likely sites for interesting phenomena such as scintillation screens. It is in these regions that the kinematic differences between neighboring clouds are most extreme and the effects of any interaction most prominent. For example, shearing flows between clouds with just moderately different velocity vectors may induce turbulence in the interaction zone between the clouds. In addition, the edges of the clouds should have higher ionization levels, as compared to the bulk of the cloud, due to the lack of significant shielding from ionizing radiation sources such as the B-star $\epsilon$ CMa and the hot white dwarfs Feige 24, HZ 43, and G191-B2B (Vallerga 1998). These regions are prime locations for enhanced electron density and high levels of turbulence and are therefore ideal sites for local scintillation screens.
The distribution of the 15 dynamical clouds in Galactic coordinates is shown in Figure 19 of Redfield \& Linsky (2008). We plot in Figure $130^{\circ}$ circular regions centered on the three quasars, showing the boundaries of nearby clouds that could be the sites of scattering screens. In Figure 2, regions where several clouds lie along the line of sight are colored, and the shading is related to the magnitude of the maximum velocity difference between any pair of clouds along that particular sight line. Since we know the three-dimensional velocity vector for all 15 clouds, we can decompose the velocity into transverse $\left(v_{1}\right.$ and $\left.v_{b}\right)$ and radial $\left(v_{r}\right)$ components. The differential velocity shown in Figure 2 is calculated from the differences between two clouds along a line of sight, $\left[\left(\Delta v_{r}\right)^{2}+\left(\Delta v_{1}\right)^{2}+\left(\Delta v_{b}\right)^{2}\right]^{1 / 2}$. All three scintillation sight lines discussed in this work are also shown in Figure 2, along with two additional known but less well studied intrahour variables. Note that all are located in regions where multiple clouds are found and the probability of cloud interactions is high. The differential velocities in these regions are 


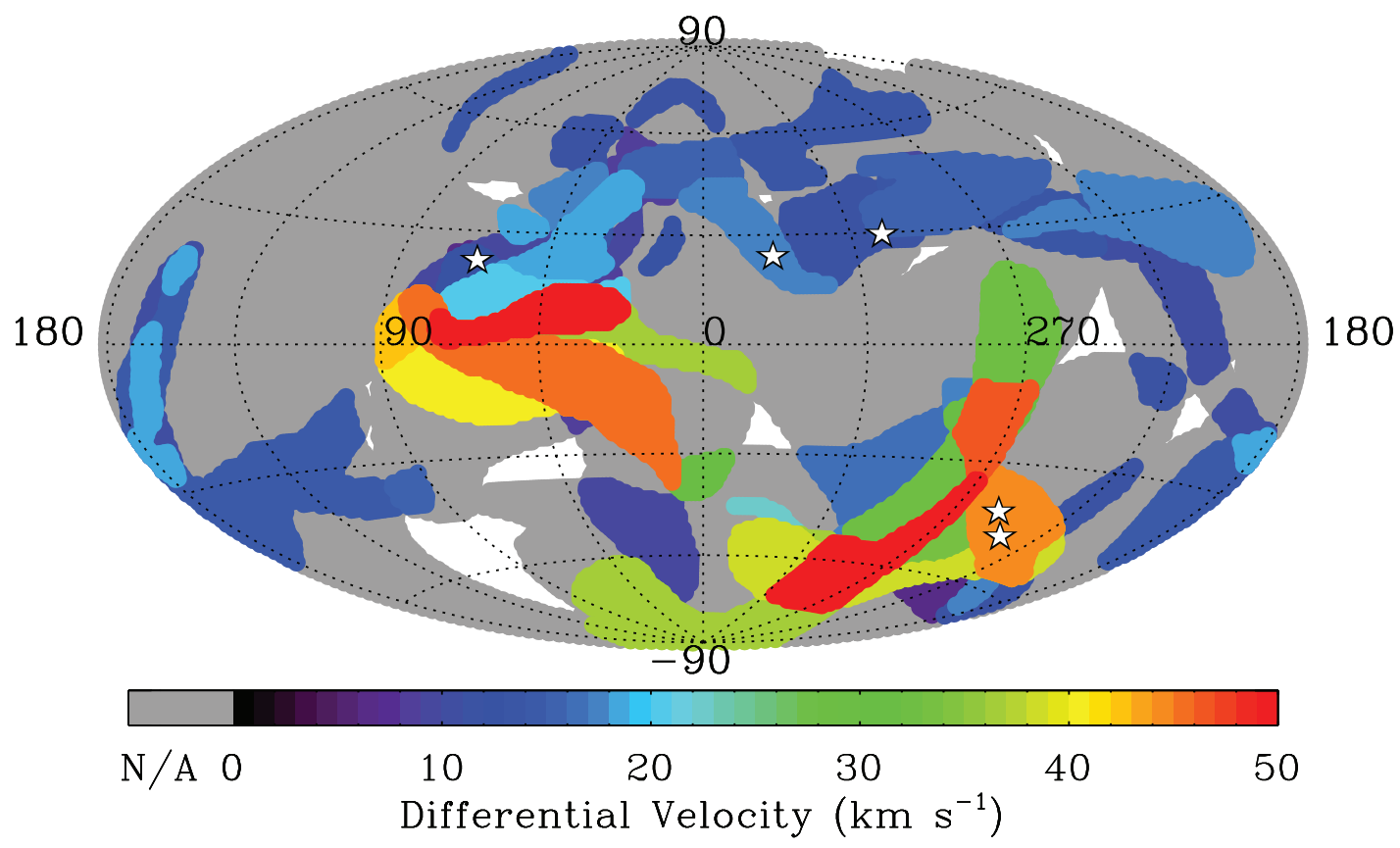

FIG. 2.-Zones of LISM cloud possible interaction. Colored regions indicate directions in which multiple LISM clouds are detected along the line of sight. The white regions indicate where no LISM cloud is detected, and gray where only a single cloud is detected. The color coding signifies the maximum magnitude of the differential velocity between clouds along the same line of sight. Five scintillation sight lines are shown here by star symbols. The three northern hemisphere sight lines (PKS B1257-326, QSO J1819+385, and QSO B1519-273) are discussed in detail in $\S 3$, while the two southern hemisphere targets (PKS 0405-385 and PSR J0437-4715) await more data. Note that all five sight lines are located in regions where multiple clouds are located and where the differential velocity can be quite high $\left(>10 \mathrm{~km} \mathrm{~s}{ }^{-1}\right.$, and in two cases $>45 \mathrm{~km} \mathrm{~s}^{-1}$ ). If the clouds are in contact with each other, such high differential velocities are likely to induce shear flows and increase local turbulence.

also significant ( $>10 \mathrm{~km} \mathrm{~s}^{-1}$, and even as high as $\sim 45 \mathrm{~km} \mathrm{~s}^{-1}$ ). The location of the scintillation sight lines is quite suggestive, but a more stringent test of the connection between scintillation screens and the warm LISM clouds can be achieved using the recently determined velocity vectors of the LISM clouds. The transverse velocity can now be calculated and directly compared to the transverse flow measured toward local scintillation screens. Without a velocity vector, the only observable velocity is the radial component. The velocity components of LISM clouds located along or near the three scintillation sight lines studied in this work are listed in Table 1. The comparison of scintillation transverse velocities with the transverse velocities of nearby warm LISM clouds is the subject of the following sections.

\section{FITTING TO THE SCINTILLATION TIMESCALE}

Observers have characterized the scintillation in flux density by forming its autocorrelation function and defining a timescale as the time lag where the correlation falls to half of its (noisecorrected) value at zero lag. When this timescale is plotted against day of year, an annual variation can be seen for the IHV sources (see Figs. 3-5). The IHV itself and the annual variation of its timescale are produced by the Earth's motion relative to the

TABLE 1

Warm LiSM Cloud Kinematics Along Radio Scintillation Sight Lines

\begin{tabular}{|c|c|c|c|c|c|c|}
\hline \multirow{3}{*}{ PKS B1257-326 ...................... } & & & Gem & $-4.75 \pm 0.97$ & $-35.99 \pm 1.10$ & $0.36 \pm 0.94$ \\
\hline & & & Leo & $-9.96 \pm 1.17$ & $-21.28 \pm 1.50$ & $0.66 \pm 0.90$ \\
\hline & & & $\mathrm{G}$ & $-17.45 \pm 1.11$ & $-23.73 \pm 1.34$ & $-2.96 \pm 1.26$ \\
\hline \multirow[t]{5}{*}{ QSO J1819+385 „.................. } & 66.2 & 22.5 & LIC & $-13.05 \pm 1.26$ & $6.54 \pm 1.36$ & $18.90 \pm 1.47$ \\
\hline & & & Mic & $-19.74 \pm 1.31$ & $-0.24 \pm 1.27$ & $20.48 \pm 1.51$ \\
\hline & & & Oph & $-26.01 \pm 0.95$ & $-6.08 \pm 1.18$ & $18.08 \pm 1.23$ \\
\hline & & & $\mathrm{G}$ & $-16.10 \pm 1.03$ & $11.74 \pm 1.63$ & $21.89 \pm 1.50$ \\
\hline & & & Aql & $-34.85 \pm 1.10$ & $42.70 \pm 1.17$ & $19.90 \pm 0.96$ \\
\hline QSO B1519-273 ..................... & & & Leo & $-19.49 \pm 1.45$ & $-12.68 \pm 1.12$ & $-3.43 \pm 0.85$ \\
\hline
\end{tabular}

Note.-All velocities are heliocentric. 

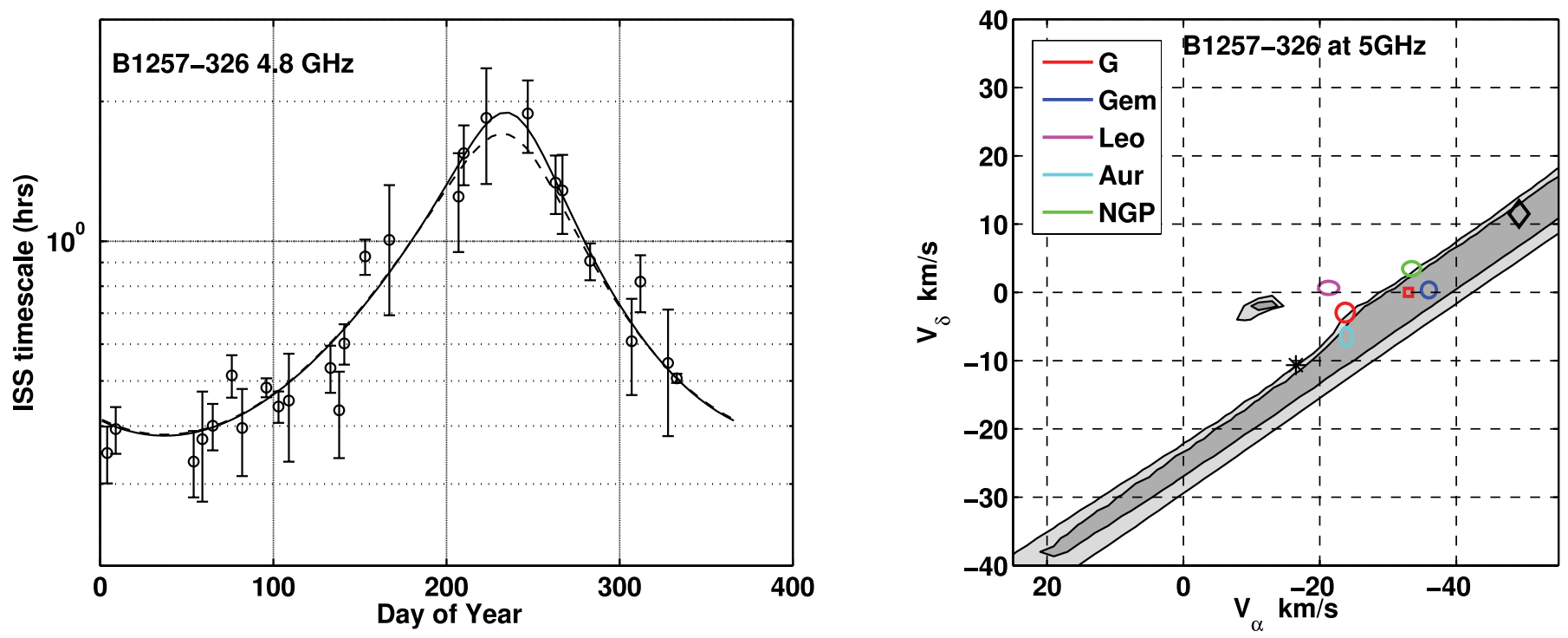

FIG. 3. - Left: Intrahour $4.8 \mathrm{GHz}$ flux variability timescale for PKS B1257-326 from Bignall et al. (2006). The solid line is the best unconstrained five-parameter fit, $V_{\mathrm{ISS}, \alpha}=-34 \mathrm{~km} \mathrm{~s}^{-1}$ and $V_{\mathrm{ISS}, \delta}=+0 \mathrm{~km} \mathrm{~s}^{-1}$ The dashed line is for a three- parameter fit assuming the transverse velocity for the Gem cloud. Right: Velocity plot for PKS B1257-326. The entire plotted region of transverse velocity space $\left(V_{\mathrm{ISS}, \alpha}, V_{\mathrm{ISS}, \delta}\right)$ was searched for the best three parameter fits to the ISS timescale data. The contours show the (reduced) $\chi^{2}$ (at 1.2 and 1.4) for three-parameter fits to the data. The error ellipses are overplotted for the transverse velocities of clouds along that line of sight. Good fits (inner contour at $\chi^{2}<1.2$ ) are obtained for the Gem and Aur clouds. The black star is for the projected transverse velocity of the local standard of rest. The best five-parameter fit (square) is very close to the transverse velocity of the Gem cloud. The diamond shows the best combined fit to the same timescale data and the ISS time delays by Bignall et al. (2006).

scintillation pattern of focused and defocused waves produced by the turbulent plasma. The timescale for interstellar scintillation, $t_{\mathrm{ISS}}$, is determined by the characteristic spatial scale of the ISS diffraction pattern divided by the transverse velocity of the Earth relative to the pattern. Two processes cause an annual variation in $t_{\text {ISS }}$ - the changing velocity of the Earth relative to the interstellar plasma and the possibility that the diffraction pattern is not statistically circular but is characterized by an ellipse with axial ratio $A$, geometric mean spatial scale $s_{\text {ISS }}$, and orientation angle of the major axis $\theta_{A}$ with respect to east (through north). The noncircular diffraction pattern could be produced either by

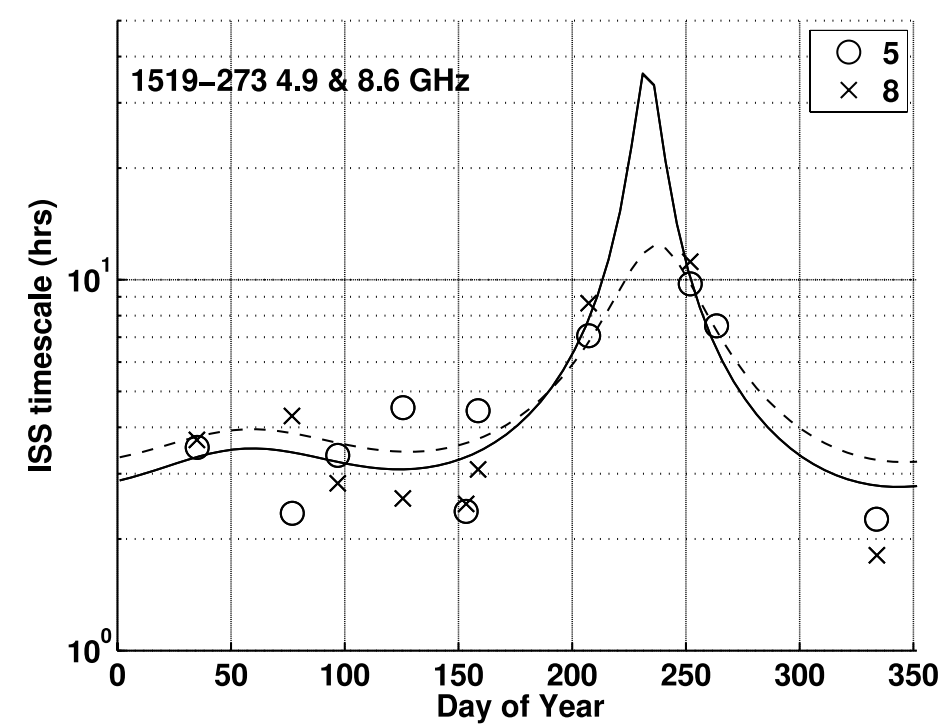

an anisotropic source structure or by anisotropic scattering. Thus both the effective pattern scale and velocity vary as the Earth orbits the Sun.

The algebraic details are as follows. If $\boldsymbol{V}_{\mathrm{E}}$ is the velocity of the Earth relative to the Sun on a given day and $\boldsymbol{V}_{\text {ISM }}$ is the (unknown) velocity of the interstellar plasma relative to the Sun, we write right ascension and declination components of the Earth's transverse velocity relative to the interstellar plasma (and to the scintillation pattern) as

$$
V_{\mathrm{ISS}, \alpha}=V_{\mathrm{E}, \alpha}-V_{\mathrm{ISM}, \alpha} ; \quad V_{\mathrm{ISS}, \delta}=V_{\mathrm{E}, \delta}-V_{\mathrm{ISM}, \delta} .
$$

FIG. 4.-Left: Intrahour 4.8 GHz flux (circles) and $8 \mathrm{GHz}$ (crosses) variability timescale data for quasar B1519-273. The solid line shows the best unconstrained five-

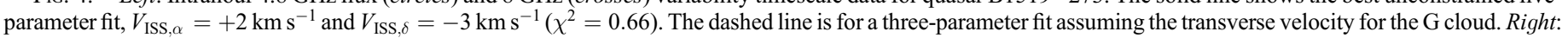

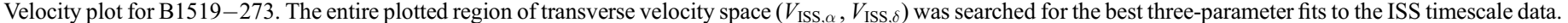

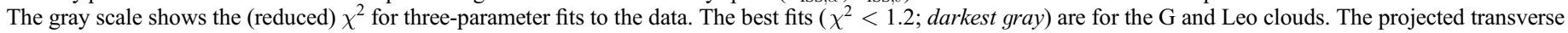
velocity of the local standard of rest (asterisk) and the best five-parameter fit (square) are not close to the transverse velocity of any cloud. 

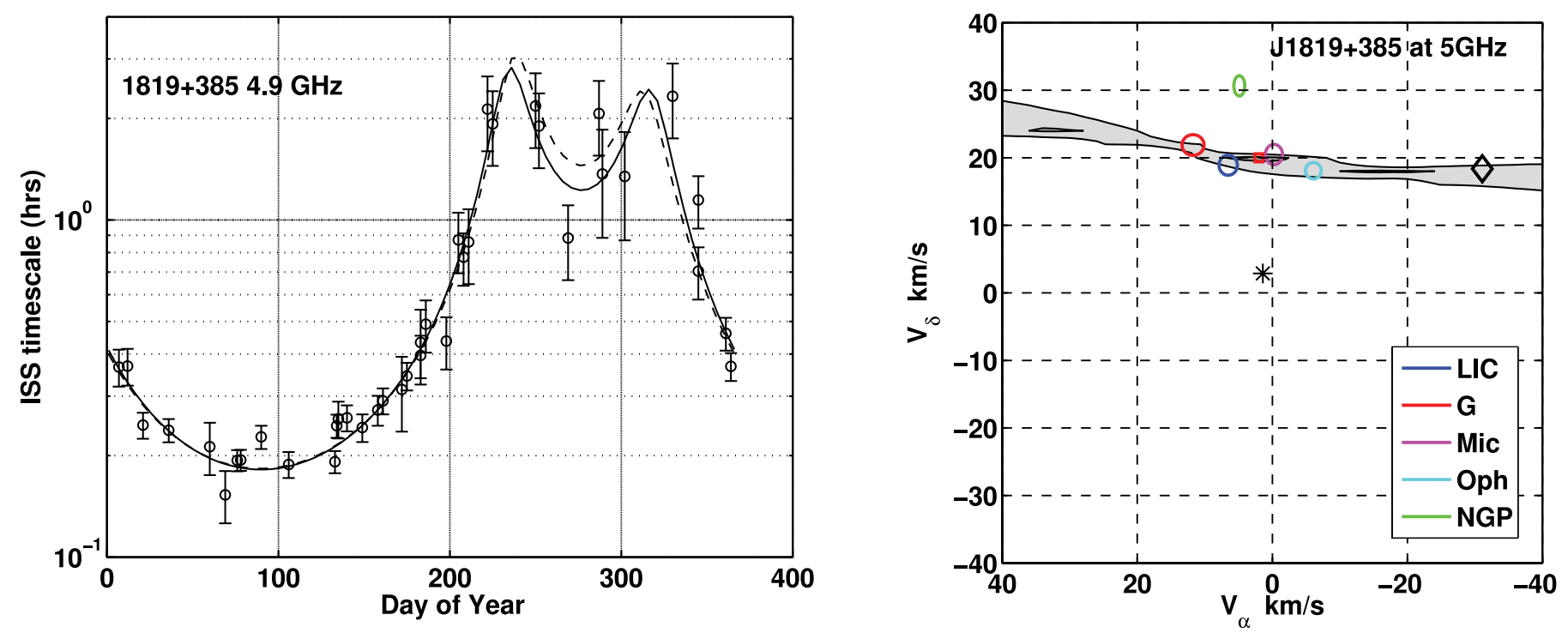

FIG. 5. - Left: Intrahour 4.9 GHz flux variability timescale data for quasar J1819+385 (Dennett-Thorpe \& de Bruyn 2003). The solid line is the best unconstrained fiveparameter fit, $V_{\mathrm{ISS}, \alpha}=+1 \mathrm{~km} \mathrm{~s}^{-1}$ and $V_{\mathrm{ISS}, \delta}=+20 \mathrm{~km} \mathrm{~s}^{-1}$. The dashed line is for a three-parameter fit assuming the transverse velocity for the Mic cloud. Right: Velocity plot for J1819+385. The entire plotted region of transverse velocity space $\left(V_{\mathrm{ISS}, \alpha}, V_{\mathrm{ISS}, \delta}\right)$ was searched for the best three-parameter fits to the ISS timescale data. The gray scale shows the (reduced) $\chi^{2}$ for three-parameter fits to the data. The best fits $\left(\chi^{2}<1.2\right)$ are for the Mic and LIC clouds, although the G and Oph clouds provide fits with reduced $\chi^{2} \approx 1.8$. The projected transverse velocity of the local standard of rest is indicated by the asterisk. The best five-parameter fit (square) is very close to the transverse velocity of the Mic cloud. The diamond indicates the transverse velocity determined by Dennett-Thorpe \& de Bruyn (2003).

Taking the spatial correlation function of the intensity scintillation to be a simple function of a quadratic form in orthogonal transverse coordinates, the resulting ISS timescale is

$$
t_{\mathrm{ISS}}=s_{\mathrm{ISS}} /\left(a V_{\mathrm{ISS}, \alpha}^{2}+b V_{\mathrm{ISS}, \delta}^{2}+c V_{\mathrm{ISS}, \alpha} V_{\mathrm{ISS}, \delta}\right)^{0.5},
$$

where for the ellipse specified as above, the coefficients of the quadratic form are $a=\cos ^{2} \theta_{A} / A+A \sin ^{2} \theta_{A}, b=\sin ^{2} \theta_{A} / A+$ $A \cos ^{2} \theta_{A}, c=2 \sin \theta_{A} \cos \theta_{A}(1 / A-A)$, and $s_{\text {ISS }}$ is the geometric mean spatial scale on which the intensity correlation falls to $1 / e$. Equation (2) is mathematically the same as equation (3) of Bignall et al. (2006).

The radio observations are typically a set of $t_{\text {ISS }}$ versus date measurements, as shown by the points in the left-hand panels of Figures 3, 4, and 5. The fitting task is to find the three ISS parameters $\left(A, \theta_{A}, s_{\mathrm{ISS}}\right)$ and the two plasma velocities $\left(V_{\mathrm{ISM}, \alpha}, V_{\mathrm{ISM}, \delta}\right)$ that give the best fit using this model equation. It can be shown that there are five independent coefficients in the general case, but that the five physical parameters depend nonlinearly on the $t_{\text {ISS }}$ data and so cannot be estimated independently. Thus, while we fitted for the best of these five parameters, we also investigated the error surface by stepping through a grid of values for $\left(V_{\mathrm{ISM}, \alpha}, V_{\mathrm{ISM}, \delta}\right)$ and at each step fitting for the remaining three ISS parameters; we constrained the axial ratio to be less than 10 , since the fit becomes degenerate for very large axial ratios, in a fashion similar to that used by Dennett-Thorpe \& de Bruyn (2003). The right-hand panels of the same figures show that for all three sources there is a satisfactory fit in an extended valley in ISM velocity space.

\subsection{Quasar B1257-326}

PKS B1257-326 is a flat spectrum, radio-loud quasar at $z=$ 1.256 that exhibits IHV at frequencies of several $\mathrm{GHz}$ (Bignall et al. 2003, 2006). Figure 3 (left) shows the ISS timescales for PKS B1257-326 observed on different days in 2000-2003 at $4.8 \mathrm{GHz}$ by Bignall et al. (2006; kindly provided by H. Bignall). The contours in the right panel show the range in transverse ve- locities that lead to fits with reduced $\chi^{2}<1.4$. With 26 observations and fitting for three parameters, the good fits correspond to $\chi^{2} \lesssim 1.2$. In their analysis of the same timescale data but also including the ISS time delays between two widely separated radio telescopes, Bignall et al. (2006) found a best combined fit at velocities $V_{\mathrm{ISS}, \alpha}=-49.2 \mathrm{~km} \mathrm{~s}^{-1}$ and $V_{\mathrm{ISS}, \delta}=11.5 \mathrm{~km} \mathrm{~s}^{-1}$, where the axial ratio was constrained to be less than 12.0. They concluded that the scintillation pattern is highly elongated with an axial ratio $A \geq 12$, and the scattering screen is located within $10 \mathrm{pc}$ of the Earth. We also find large axial ratios for the ISS pattern.

Also plotted in the figure are the transverse velocities of the five nearby clouds located close to the line of sight to the quasar. The transverse velocities for two clouds (Aur and Gem) give very good fits, and the Gem cloud has a velocity very close to the best unconstrained five-parameter fit, $V_{\mathrm{ISS}, \alpha}=-34 \mathrm{~km} \mathrm{~s}^{-1}$ and $V_{\text {ISS }, \delta}=0 \mathrm{~km} \mathrm{~s}^{-1}$. The ISS parameters for this fit are $A=4.1$, $\theta_{A}=151.3^{\circ}$, with values that vary systematically along the band of good fits in velocity space. Note also that there is a small isolated region centered on $V_{\mathrm{ISS}, \alpha}=-12 \mathrm{~km} \mathrm{~s}^{-1}$ and $V_{\mathrm{ISS}, \delta}=$ $-2 \mathrm{~km} \mathrm{~s}^{-1}$ with nearly the same minimum $\chi^{2}$ that is far from any cloud velocities.

The diamond indicates the transverse velocity obtained by Bignall et al. (2006), which is well outside the error bounds for the cloud velocities. They point out that the large anisotropy of the scintillation pattern permits a range of best fit velocities that lie along a straight line in the $\left(V_{\mathrm{ISS}, \alpha}, V_{\mathrm{ISS}, \delta}\right)$ plane. This line is situated along the best-fit line in Figure $3(r i g h t)$. Their best-fit value of $V_{\mathrm{ISS}, \alpha}=-49.2 \mathrm{~km} \mathrm{~s}^{-1}$ and $V_{\mathrm{ISS}, \delta}=11.5 \mathrm{~km} \mathrm{~s}^{-1}$ is therefore poorly constrained. However, it is remarkable that four of the five clouds have transverse velocities in the band of velocities that provide "reasonable" fits to the ISS data. We show the timescale fit for the closest fitting Gem cloud by the dashed line in the left panel of the figure. The clouds in this group are located within $\pm 10 \mathrm{~km} \mathrm{~s}^{-1}$ of each other, suggesting a real physical association between these clouds and the plasma producing the scintillation. 
Although the best fits (lowest $\chi^{2}$ ) in Figure 3 are for both the Aur and Gem clouds, the line of sight to the quasar passes through the edge of the Gem cloud but is about $15^{\circ}$ outside of the Aur cloud (Fig. 1). For this reason, we believe that the scintillation screen lies at the edge of the Gem cloud, which lies within $6.7 \mathrm{pc}$ of the Sun (Redfield \& Linsky 2008). The orientation of the edge of the Gem cloud in celestial coordinates is about $180^{\circ}$, compared to $\theta_{A}=151.3^{\circ}$.

\subsection{Quasar B1519-273}

The 5 and $8 \mathrm{GHz}$ timescale data from Jauncey et al. (2003) in the left panel of Figure 4 are overplotted as in their Figure 1. These data are not as extensive as for the other two sources, and there are no errors given for the timescales. Since the data do not show a consistent difference in timescale between the two frequencies, we fit the two data sets simultaneously. The right panel (same format as for B1257-326) shows the contours that define a good fit and the error ellipses for the four clouds near that line of sight. Both the $G$ and Leo clouds give good fits, lying within the $\chi^{2}=1.2$ contour. We note that in the absence of error bars for the timescales, the estimate of $\chi^{2}$ is based on the goodness of fit, so that its normalization is not reliable. The timescale model for the $\mathrm{G}$ cloud is overplotted in the left panel together with the best five-parameter fit- $V_{\text {ISS }, \alpha}=-1.7 \mathrm{~km} \mathrm{~s}^{-1}, V_{\mathrm{ISS}, \delta}=-3.4 \mathrm{~km} \mathrm{~s}^{-1}$. The ISS parameters for this fit are $A=3.5, \theta_{A}=168^{\circ}$. We note that these velocities are distinct from that of the local standard of rest used by Jauncey et al. (2003) in their analysis of the same timescale data. We conclude that the scintillation screen lies near the edge of the $\mathrm{G}$ cloud, since the Leo cloud lies about $20^{\circ}$ from the source (Fig. 1). The inner edge of the G cloud lies closer than $1.3 \mathrm{pc}$ from the Sun and may extend several parsecs away from the inner edge. The orientation of the $\mathrm{G}$ cloud edge in celestial coordinates is about $110^{\circ}$, compared to $\theta_{A}=168^{\circ}$.

\subsection{Quasar J1819+385}

Figure 5 (left) shows the excellent $5 \mathrm{GHz}$ ISS data overplotted from the years 1999-2001 for the quasar J1819+385 observed by Dennett-Thorpe \& de Bruyn (2003). This source shows the largest intraday variability presently known at radio wavelengths (Dennett-Thorpe \& de Bruyn 2001). The best five-parameter fit to these data is for $V_{\mathrm{ISS}, \alpha}=-1 \mathrm{~km} \mathrm{~s}^{-1}$ and $V_{\mathrm{ISS}, \delta}=+20 \mathrm{~km} \mathrm{~s}^{-1}$, which is within the uncertainty of the Mic cloud velocity.

The right panel shows the narrow band in transverse velocity space corresponding to fits to the ISS data with reduced $\chi^{2}<1.2$. The transverse velocities of the Mic and LIC clouds are the best fits to the ISS data, although the G and Oph clouds provide fits with reduced $\chi^{2} \approx 1.8$. Again, four of the six clouds have transverse velocities that give good-to-reasonable fits to the ISS data. The transverse velocity of the local standard of rest is an unacceptable fit to the data, as already reported by Dennett-Thorpe \& de Bruyn (2003).

Our analysis, which follows that of Dennett-Thorpe \& de Bruyn (2003), agrees with their result that the ISS data lie in a narrow region of velocity space, as shown in Figure 5 (right). By combining their timescale data with time delay data on an intercontinental baseline, they arrived at a best fit for $V_{\mathrm{ISS}, \alpha}=-33.5 \mathrm{~km} \mathrm{~s}^{-1}$ and $V_{\mathrm{ISS}, \delta}=+13.5 \mathrm{~km} \mathrm{~s}^{-1}$ relative to the local standard of rest. This is plotted with the diamond in the heliocentric reference system that we use in this paper. Their result is well removed from the values for the local clouds, but as stated in a footnote in their paper, Dennett-Thorpe \& de Bruyn (2003) used an algorithm for interpreting the time delay data that was not corrected for anisotropic scintillation. We therefore make no further use of that value plotted by the diamond. Their Figures 10 and 11 give results for fitting to the annual changes in timescale that yield an axial ratio $A>6$ oriented at $\theta_{A}=7^{\circ} \pm 4^{\circ}$ This is consistent with our result, which gives a best fit at $A=11, \theta_{A}=5.5^{\circ}$. They also conclude that the thin-scattering screen is located only $1-12 \mathrm{pc}$ from the Sun.

The line of sight to the quasar lies well inside the LIC and close to the edge of the Mic cloud, the best fits in Figure 5. It also lies close to the edge of the $\mathrm{G}$ cloud but some $5^{\circ}$ outside of the Oph cloud. We believe that the most likely candidate cloud for the scintillation source is the Mic cloud since Redfield \& Linsky (2008) show that this cloud appears to be compressed by the LIC and $\mathrm{G}$ clouds, and the Mic cloud has the highest temperature $(9900 \pm 2000 \mathrm{~K})$ of the 15 clouds they studied. The Mic cloud lies within $5.1 \mathrm{pc}$ of the Sun. The orientation of the Mic cloud edge in celestial coordinates is about $30^{\circ}$ compared to $\theta_{A}=5.5^{\circ}$.

\section{CONCLUSIONS}

We have fit the ISS timescale observations of the three quasars that show the largest amplitude intraday variations and have highquality data sets. For each quasar, our search of a large range of possible transverse velocities of the scintillation screen yielded good agreement with the annular variation of the ISS data for a relatively narrow range of transverse velocities. For each quasar there are at least two warm interstellar clouds that have transverse velocities consistent with the acceptable range (reduced $\chi^{2} \leq$ 1.2 ), but we propose that the scattering screen is located in one of the clouds near its edge. The transverse velocity of the local standard of rest, the usual assumption in previous studies, is an unacceptable solution for all three quasars.

In all cases, the scintillation screen is asymmetrical. We compared the ellipse orientations $\theta_{A}$ with the orientations of the nearest cloud edge to see whether the turbulence structure is elongated parallel to the cloud edge, perhaps by a magnetic field confining the outside of the cloud. We found no preferred alignments as the ellipse orientations differ typically by $25^{\circ}-50^{\circ}$ from the orientation of the edge of the cloud that we believe is the location of the scattering screen. This apparent misalignment may indicate that the cloud edges have complex geometries that our coarse fitting to a modest number of lines of sight cannot reveal. Also Rickett (2007) and Ramachandran et al. (2006) show that the turbulent regions producing the observed scintillation are likely very small on the basis of the arrival times of pulses from the millisecond pulsar B1937+21 and other arguments.

Previous authors have placed the locations of the scattering screens near to the Sun on the basis of the scintillation timescales and the time delays between the scintillation flux variations as seen by two widely separated radio telescopes. Our analysis confirms and places more stringent constraints on the distances to the scattering screens. For example, Bignall et al. (2006) place the scattering screen toward B1257-326 within 10 pc of the Sun, and we argue that it is located within $6.7 \mathrm{pc}$. Dennett-Thorpe \& de Bruyn (2003) argue that the scattering screen for J1819+385 is located between 1 and 12 pc from the Sun, and Macquart \& de Bruyn (2007) present two models that fit their data with the scattering screen located at $3.8 \pm 0.3 \mathrm{pc}$ or $2.0 \pm 0.3 \mathrm{pc}$. We argue that the screen toward this source is located at the edge of the Mic cloud within $5.1 \mathrm{pc}$ of the Sun. If the Mic cloud is indeed compressed and heated by the LIC and G clouds, then the scattering screen in the Mic cloud is located much closer to the Sun than $5.1 \mathrm{pc}$. The scattering screen toward B1519-273 near the edge of the $\mathrm{G}$ cloud is likely closer than 3 pc from the Sun.

An important result is that for each source, the line of sight to the quasar passes through or very near to at least two warm 
clouds. Although we know only upper limits to the distances to most of these clouds, they are all nearby and could interact. For example, interaction of the LIC and G clouds is likely responsible for the shape and high temperature of the Mic cloud. Since typical velocity differences between clouds along the three lines of sight are $10-20 \mathrm{~km} \mathrm{~s}^{-1}$, the interactions could easily produce turbulence that would be responsible for the scintillation. Also, cloud edges are regions of high ionization because of the absence of shielding from the strong UV radiation from hot stars and white dwarfs. Figure 2 shows areas on the sky where the velocity differences between clouds along the line of sight are large and where cloud-cloud interactions could produce turbulence. We predict that these are regions where large-amplitude IDV sources will be discovered. Two examples that deserve further study are the two IDV sources located in the southern hemisphere (PKS 0405-385 and PSR J0437-4715) that are plotted in Figure 2 but are not analyzed in this paper as highquality ISS data are not yet published.

We thank Hayley Bignall for providing the data on PKS B1257-326 that we have analyzed in this paper. We also thank Jean-Pierre Macquart for discussions on this topic and the referee for his very useful suggestions. B. R. thanks the NSF for support under grant AST 05-07713. S. R. would like to acknowledge support provided by NASA through Hubble Fellowship grant HSTHF-01190.01 awarded by the Space Telescope Science Institute, which is operated by the Association of Universities for Research in Astronomy, Inc., for NASA, under contract NAS 5-26555.

Bignall, H. E., Macquart, J.-P., Jauncey, D. L., Lovell, J. E. J., \& Tzioumis, A. K. 2006, ApJ, 652, 1050

Bignall, H. E., et al. 2003, ApJ, 585, 653

Dennet-Thorpe, J., \& de Bruyn, A. G. 2001, Nature, 415, 67 2003, A\&A, 404, 113

Frisch, P. C., Grodnicki, L., \& Welty, D. E. 2002, ApJ, 574, 834

Heeschen, D. S., Krichbaum, T., Schalinski, C. J., \& Witzel, A. 1987, AJ, 94, 1493

Jauncey, D. L., Johnston, H. M., Bignall, H. E., Lovell, J. E. J., KedzioraChudczer, L., Tzioumis, A. K., \& Macquart, J.-P. 2003, Ap\&SS, 288, 63

Lallement, R., \& Bertin, P. 1992, A\&A, 266, 479

Lallement, R., Ferlet, R., Lagrange, A. M., Lemoine, M., \& Vidal-Madjar, A. 1995, A\&A, 304, 461

Lallement, R., Welsh, B. Y., Vergely, J. L., Crifo, F., \& Sfeir, D. 2003, A\&A, 411,447

REFERENCES

Lovell, J. E. J., et al. 2007, in ASP Conf. Ser. 365, SINS - Small Ionized and Neutral Structures in the Diffuse Interstellar Medium, ed. M. Haverkorn \& W. M. Goss (San Francisco: ASP), 279

Macquart, J.-P., \& de Bruyn, A. G. 2007, MNRAS, 380, L20

Quirrenbach, A., et al. 1992, A\&A, 258, 279

Ramachandran, R., Demorest, P., Backer, D. C., Cognard, I., \& Lommen, A. 2006, ApJ, 645, 303

Redfield, S., \& Linsky, J. L. 2004, ApJ, 602, 776 . 2008, ApJ, in press

Rickett, B. 2007, in ASP Conf. Ser. 365, SINS-Small Ionized and Neutral Structures in the Diffuse Interstellar Medium, ed. M. Haverkorn \& W. M. Goss (San Francisco: ASP), 207

Rickett, B. J., Lazio, T. J. W., \& Ghigo, F. D. 2006, ApJS, 165, 439

Vallerga, J. 1998, ApJ, 497, 921 